\title{
Inercia e innovación en la empresa familiar: una primera aproximación
}

\section{Inertia and innovation in family firms: a first approach}

\author{
José Daniel Lorenzo Gómez ${ }^{\mathrm{a}, *} \cdot$ Pedro Núñez-Cacho Utrilla ${ }^{\mathrm{b}}$ \\ ${ }^{a}$ Departamento de Organización de Empresas. Universidad de Cádiz, 11002 (Spain) \\ ${ }^{b}$ Departamento de Organización de Empresas, Marketing y Sociología. Universidad de Jaén, 23071 (Spain)
}

D A T OS ARTÍCULO

Historial:

Recibido 09-07-2012

Aceptado 16-11-2012

Palabras clave:

Empresa familiar

Innovación

Obstáculos

Inercias

Códigos JEL:

015

A R T I C L E IN F O

Article history:

Received 09-07-2012

Accepted 16-11-2012

Keywords:

Family Business

Innovation

Obstacles

Inertia

JEL codes:

015

\section{RES U M EN}

Las particularidades de la innovación en el ámbito de la empresa familiar suponen un aspecto aun escasamente desarrollado en los estudios sobre estas organizaciones $\mathrm{y}$, más específicamente, existen numerosas cuestiones por resolver en relación con las barreras a la innovación en la empresa familiar. La aplicación del modelo de las cinco fuerzas inerciales de Rumelt (1995) al caso concreto de la empresa familiar permite hacer una primera aproximación al estudio de los obstáculos que dificultan la innovación en la empresa familiar. Del análisis realizado se derivan una serie de proposiciones, como punto de partida para una investigación más amplia.

\section{A B S T R A C T}

The study of specific features of innovation in the family business is poorly developed and, more specifically, there are numerous unresolved issues regarding barriers to innovation in the family business. Applying the five inertial forces model by Rumelt (1995) to the case of the family business is provided an initial approach to the study of barriers to innovation in the family business. This analysis has derived a series of propositions, as a starting point for subsequent investigation.

\footnotetext{
* Autor de contacto.

Correoselectrónicos: daniel.lorenzo@uca.es,pnunez@ujaen.es
} 


\section{Introducción}

La innovación comprende el proceso de desarrollo y aplicación de una nueva idea (Van de Ven, Polley, Garud y Venkataraman, 2001). Según Varis y Littunen (2010) es el elixir de la vida para las empresas, independientemente de su tamaño u otros atributos de la organización, ya que el crecimiento, el éxito y la supervivencia de la empresa depende de su capacidad de innovación continua a lo largo del tiempo, especialmente en mercados inestables e inciertos.

La noción de innovación va más allá de la idea de invención referida a la creación de nuevo conocimiento. Puede basarse en conocimiento nuevo o bien en la reconfiguración de forma novedosa del existente (Schumpeter, 1934; Drucker, 1985). Desde un punto de vista económico, para que un producto, un servicio o un proceso productivo se pueda considerar como una innovación, no tiene por qué ser algo nuevo en sentido estricto, sino nuevo en el mercado en dónde se quiere introducir (Koellinger, 2008). En consecuencia, la innovación es un concepto subjetivo, por lo que la condición de innovadora aplicada a una actividad depende de la perspectiva del observador, tanto desde el punto de vista del conocimiento empleado en la innovación como desde una visión económica. La innovación se puede referir a nuevos productos, nuevos procesos y nuevas formas de gestión. Sea cual sea la forma que revista, implica un proceso de cambio en la empresa.

Pese a su trascendencia, la innovación en la empresa familiar es un aspecto aun escasamente estudiado y sobre el que quedan por responder numerosas cuestiones, Craig y Moores, (2006) sugieren que las empresas familiares no tienen por qué ser más adversas al riesgo ni menos innovadoras que las empresas no familiares, considerando que conforme evolucionan en el tiempo pueden ser incluso más innovadoras que en sus inicios. Otros estudios sin embargo indican que, con el paso del tiempo se vuelven conservadoras y no quieren o no pueden asumir los riesgos asociados a la actividad emprendedora (Autio y Mustakallio, 2003; Zahra, Hayton y Salvato, 2004). Además, los fundadores de empresas familiares, que desean construir un legado duradero, pueden volverse más conservadores en sus decisiones debido al riesgo elevado de fracaso de un proyecto empresarial, así como por el riesgo de destrucción de la riqueza familiar (Sharma, Chrisman, y Chua, 1997; Zahra et al., 2004). Uno de los tópicos sobre empresa familiar es que tiene un carácter más conservador respecto a la innovación en comparación con las no familiares (Bermejo, 2008; Tàpies, 2009). Sin embargo, no se han aportado argumentos solventes que ratifiquen o desmientan esta idea e incluso autores como Aronoff (1998) han indicado que pueden ser muy innovadoras y agresivas en sus mercados.

$\mathrm{Si}$ bien son escasos los estudios sobre innovación en la empresa familiar (Craig y Moores, 2006), el análisis de los obstáculos específicos a la innovación en la empresa familiar es un aspecto que está particularmente inexplorado, y sobre el que se centra el presente estudio. Por tanto, se precisa un mayor esfuerzo por parte de los académicos que trabajan en esta materia para avanzar en el conocimiento de los aspectos específicos de la innovación en la empresa familiar para llegar a conclusiones sólidas. Para contribuir a cubrir esta laguna en los estudios sobre empresa familiar, en este artículo se propone la aplicación del modelo de las fuerzas inerciales de Rumelt (1995), con el objetivo de analizar las dificultades del proceso de cambio que desencadena la innovación en el ámbito específico de las empresas familiares.

Este trabajo se centra en el caso concreto de la empresa familiar, al entender que existen factores que hacen que su gestión de la innovación sea diferente al resto de organizaciones. Por regla general, los investigadores en este campo consideran que es la influencia de la familia o familiness (Habbershon y Williams, 1999; Nuñez-Cacho y Grande, 2012) el factor que hace que este tipo de empresas sean distintas al resto. Ahora bien, para poder concluir que esto es realmente cierto, es preciso identificar la naturaleza de esas diferencias y determinar cómo y porqué afectan al desempeño de la familia empresaria. Hoffman et al. (2006) indican que el ambiente de trabajo 
de las empresas familiares es único, lo que proporciona una mayor motivación y más eficiencia en sus prácticas $\mathrm{y}$, por tanto, en la generación de ventaja competitiva (Levring y Moskowitz, 1993). Tagiuri y Davis (1996) coinciden en que el ambiente familiar propicia una mayor motivación, lealtad y confianza. Sirmon y Hitt (2003) señalan que los recursos humanos forman parte del capital distintivo de la empresa familiar y su gestión es más complicada que en el resto de empresas, debido a la dualidad de las relaciones existentes.

Las aportaciones de Cabrera, De Saá y García (2001) y Larraza et al. (2007), señalan la existencia de diferencias estratégicas entre las empresas familiares y no familiares motivadas por la divergencia entre los objetivos de ambas, por los valores de la familia, por su influencia en el negocio y por el hecho de que algunos empleados sean, a su vez, miembros de la familia. En la misma dirección, Habbershon et al. (2003) miden, desde la perspectiva teórica de recursos y capacidades y la teoría de sistemas, la influencia de la familia en la empresa a través del familiness, denominación que recoge el efecto que provoca la interacción entre los sistemas familia y empresa y que ayuda a conseguir una posición ventajosa respecto a la competencia. Este efecto crea en la empresa familiar un ambiente único que hace que su comportamiento sea distinto al resto. Para Chrisman, Chua y Litz (2003) el familiness contribuye a la creación de una posición competitiva añadiendo beneficios no económicos a la organización, ya que consideran la empresa familiar como un conjunto sinérgico de recursos único debido a las interacciones que existen entre familia $y$ empresa.

Así pues, el objetivo del presente trabajo es conocer las inercias propias de las empresas familiares, derivadas de su carácter familiar y que actúan como freno u obstáculo a su capacidad para innovar. Para ello esta investigación se estructura en cuatro secciones, además de este apartado introductorio. En primer lugar, se plantea el marco teórico y se revisa la literatura relacionada con la innovación en la empresa familiar. A continuación, se expone el modelo de las cinco fuerzas de Rumelt (1995), que explica las inercias al cambio en las organizaciones, y se aplica a la empresa familiar. De esta aplicación surgen una serie de proposiciones, cuya contrastación excede el espacio del presente artículo y que serán objeto de estudio en una fase posterior de esta investigación. Por último, se ofrece un apartado de discusión y conclusiones, terminando con las limitaciones y la propuesta de líneas futuras de investigación.

\section{Marco teórico}

\section{1 El enfoque de recursos y capacidades.}

La visión de la empresa basada en los recursos (RBV) (Wernerfelt, 1984; Grant, 1991; Barney, 1991) se utiliza de forma creciente como marco teórico para la investigación sobre empresa familiar (Sharma et al. 1997; Cabrera, De Saá y García, 2001; Sirmon y Hitt, 2003; Moores, 2009; Debicki et al., 2009) y a partir de este enfoque se han desarrollado conceptos como el de familiness (Habbershon y Williams, 1999) en referencia a la dotación específica de recursos en la empresa familiar, que está condicionada por la interrelación entre familia y empresa.

Este enfoque es uno de los más utilizados en los últimos años para identificar los recursos y capacidades que hacen que la empresa familiar tenga un potencial único y muestra cómo desarrollarlo para obtener ventajas competitivas basadas en su carácter familiar (Habbershon et al., 2003; Habbershon y Williams, 1999). Su base es la influencia que la familia ejerce en la empresa, por ello, una de las cuestiones más importantes para la mayoría de los autores es determinar si el "efecto familia" supone un recurso o, por el contrario, supone un coste (Dyer, 2006).

Son mucho más frecuentes los estudios sobre los aspectos positivos que, por sus particularidades, presentan las empresas familiares. Sin embargo, además de estas diferencias que afectan positivamente al éxito de la empresa familiar, se encuentran una serie 
de factores que pueden incidir de manera negativa en su rendimiento $\mathrm{y}$, por tanto, lejos de ser una fuente donde obtener ventajas competitivas, suponen un peso o lastre que la empresa familiar ha de superar. En este sentido Kellermanns (2005) manifiesta que los recursos y capacidades de las empresas familiares son el resultado de la influencia del sistema familiar en el empresarial y que ambos sistemas deben ser dirigidos cuidadosamente para que puedan generar una influencia positiva. Además, explica que los distintos tipos de estructura y cultura de la familia empresaria tienen influencias positivas y negativas en la acumulación de recursos y varían según las características de cada empresa familiar.

También Dyer (2006) señala que las diferentes características que puede presentar la empresa familiar influyen sobre la gestión de los recursos de la empresa, por lo que considera necesario establecer una clasificación de los diferentes tipos de familia para ver los efectos positivos o negativos de la influencia familiar en cada una de ellas. Otros autores como Aldrich y Cliff (2003), Haynes et al. (1999) o Tocarczyk et al. (2007), no conformes con la afirmación anterior, manifiestan que la influencia de la familia en la gestión de los recursos tiene siempre un enfoque positivo.

Por otro lado, la RBV señala que la mera posesión de recursos valiosos, raros, difíciles de imitar e integrados en la organización (Barney y Griffin, 1992) no supone automáticamente la consecución de ventajas competitivas (Sirmon y Hitt, 2003), sino que requiere además una adecuada gestión de estos recursos por parte de la dirección de la empresa (Chirico y Nordqvist, 2010). Esto lleva a una forma de gestión que, particularmente en el caso de empresas familiares, facilite la incorporación de nuevos recursos, la renovación de los existentes, su integración con otros recursos y capacidades y su apalancamiento en la organización (Sirmon y Hitt, 2003).

Esta forma de gestión de los recursos se relaciona con la noción de capacidades dinámicas (Teece, Pisano y Shuen, 1997) orientada a la gestión del cambio, al impulso emprendedor y a la creación de valor a lo largo del tiempo (Chirico y Nordqvist, 2010). En el caso de las empresas familiares, tiene especial interés conocer cómo se produce la transmisión de conocimiento y valores, para poder entender el modo en que se renuevan los recursos, que permite no sólo la supervivencia, sino además el crecimiento y desarrollo del negocio original a lo largo de las sucesivas generaciones (Sirmon y Hitt, 2003; Chirico y Nordqvist, 2010). Estas ideas enlazan con lo planteado por Cabrera et al. (2001) y Steier (2001), que han tratado la cuestión de la transferencia de recursos a través de las distintas generaciones y su importancia para la continuidad de la empresa familiar, señalando que la transmisión de conocimiento dentro de la familia es una de las claves que contribuirá al desarrollo de la ventaja competitiva de la misma y que aumentará el factor positivo del familiness. Por ello, es necesario conservar el conocimiento tácito dentro de la empresa y trasmitirlo a los futuros directivos para la construcción de una fuerte cultura corporativa (Miller y Le Breton-Miller, 2006).

Analizando y corrigiendo las limitaciones provocadas en la empresa familiar por el "familiness negativo" y que afectan al ámbito de la innovación, se podrán aplicar medidas correctoras para que el carácter familiar de la empresa, su capacidad para la trasmisión de conocimiento y sus capacidades dinámicas propias no sean una carga, sino una ventaja competitiva sostenible.

\subsection{Innovación y empresa familiar}

Como es sabido, no existe una definición de empresa familiar universalmente aceptada. Gallo (1995) considera que existen tres puntos en común en las múltiples definiciones de empresa familiar propuestas: la propiedad, en referencia al porcentaje de acciones o control efectivo de la empresa; la gestión, entendida como el desempeño de tareas ejecutivas en la empresa y la capacidad para nombrar ejecutivos, normalmente personas de la familia; y la continuidad, que se refiere al número de generaciones familiares implicadas en la gestión de la empresa. 
En Europa, las asociaciones nacionales de empresas familiares han adoptado como definición formal la propuesta por el Grupo Europeo de Empresa Familiar (GEEF), en 2008, que considera empresa familiar aquella en la que:

1. la mayoría de los votos son propiedad de la persona o personas de la familia que fundó o adquirió la compañía;

2. la mayoría de los votos puede ser directa o indirecta;

3. al menos un representante de la familia participa en la gestión o gobierno de la compañía;

4. las compañías cotizadas se consideran como empresa familiar si la persona que fundó o adquirió la compañía, o sus familiares o descendientes poseen el $25 \%$ de los derechos de voto a los que da derecho el capital social.

La interrelación entre empresa familiar y el desarrollo de proyectos emprendedores ha sido analizada en diferentes estudios (Hoy y Verser, 1994; Veciana, 1999; Aldrich y Cliff, 2003; Chrisman et al., 2003; Chua, Chrisman y Steier, 2003; Zahra et al., 2004; Steier, 2007; Steier, 2009). Rogoff, Kay y Heck (2003; p. 559) destacan que "la familia es el oxígeno que alimenta la llama de la capacidad de emprender". Casado y Rodríguez (2009) señalan el cultivo del espíritu emprendedor como una de las claves de la orientación a largo plazo que es más característica en las empresas familiares.

Otros trabajos, como el de Steier (2003; pp. 597-598) estudian la importancia de los familiares como apoyo al emprendedor, explicando que la implicación de la familia en el proyecto empresarial de uno de sus miembros es continua y puede ir desde la forma más generosa de altruismo hasta el egoísmo del mercado. También se ha abordado el papel de los miembros de la familia como business angels (Erikson, Sørheim y Reitan, 2003) o el soporte realizado por los miembros de la familia en la creación de empresas, para concluir que uno de cada cuatro contactos básicos en la red de un emprendedor es un familiar, con independencia de que trabaje o no en la empresa fundada por éste (Anderson, Jack y Dodd, 2005).

El carácter innovador de la empresa familiar ha sido analizado por Craig y Moores (2006), destacando que la empresa familiar concede gran importancia a la innovación como componente clave en su estrategia, a lo largo del tiempo, en un sentido comparable a las empresas que operan en sectores de tecnologías avanzadas. Estos autores indican que la investigación sobre innovación se ha centrado en las empresas grandes, que cotizan en bolsa, ignorando otro tipo de empresas, como las de carácter familiar. En su estudio Craig y Moores utilizan una muestra longitudinal de empresas familiares, analizadas en dos momentos con un intervalo de 10 años de diferencia y encuentran relación entre la innovación y el ciclo de vida de la empresa, ya que las empresas familiares analizadas muestran niveles significativamente mayores de innovación en las primeras etapas de su desarrollo. Sin embargo, este impulso innovador no desaparece a lo largo del tiempo, ya que las empresas familiares consolidadas parecen conceder gran importancia a la gestión de la innovación. Estos resultados sugieren que la innovación sigue teniendo una importancia considerable para las empresas familiares, incluso para aquellas que operan en los sectores considerados más tradicionales.

Por su parte, Pittino y Visintin (2009) analizan la innovación en la pequeña y mediana empresa familiar mediante la aplicación del modelo de tipos de estrategia de Miles y Snow (1978). A partir de la consideración del comportamiento innovador en términos tanto de inputs (en referencia a las fuentes de conocimiento) como de outputs (innovación de producto, de proceso y en el modelo de negocio), obteniendo cuatro grupos de empresas que siguen estrategias de innovación diferentes:

Defensoras, las que ponen un gran énfasis en la innovación de procesos, para reforzar una posición dominante en su sector de actividad basada en una gama de productos consolidados, con mayor peso de las relaciones externas (clientes, proveedores) frente a la generación interna de conocimiento como fuentes para la innovación. Los autores señalan 
que dentro de esta tipología se encuentran las empresas familiares de segunda o tercera generación.

- Prospectoras, con orientación hacia la innovación de productos y la exploración de nuevas áreas de negocio, buscando complementar el conocimiento internamente desarrollado (tanto por directivos familiares como por empleados no familiares) con la búsqueda de conocimiento externo a través de socios tecnológicos o de redes de difusión de conocimiento. En este grupo parece encajar la empresa familiar en primera generación, aún no implicada en el proceso de sucesión, con un impulso innovador liderado por el emprendedor que puso en marcha la empresa.

- $\quad$ Analíticas, con un perfil intermedio en innovación de producto como de procesos, equilibradas entre la explotación del modelo de negocio actual y la exploración de formas de negocio futuras, buscando la adaptación del conocimiento nuevo a las necesidades del cliente; aquí se situarían las empresas familiares en segunda o tercera generación y con la propiedad no muy concentrada, con características similares a las prospectoras.

- Reactivas, sin una orientación clara hacia la innovación, posiblemente por falta de una orientación estratégica definida, que puede causar que los esfuerzos en innovación no tengan buenos resultados. Aquí se encuentran empresas familiares inmersas en una situación de cambio, o que están pasando por dificultades para integrar los intereses de los diferentes grupos de interés en una estrategia coherente.

El trabajo de Sciascia, Chirico y Mazzola (2010) muestra que la orientación emprendedora en la empresa familiar afecta de manera positiva a los resultados si el número de generaciones implicadas en la gestión de la empresa es uno o dos, y de forma negativa si hay más de tres generaciones en la dirección que coinciden en el tiempo, debido a la posible incidencia de conflictos intergeneracionales. También es destacable la aportación de Chirico y Nordqvist (2010) analizando el impacto de la cultura organizativa en la empresa familiar, como impulsor de la orientación emprendedora $y$ también como posible inercia cuando aparece en su vertiente más paternalista.

Como se ha expuesto en la revisión de la bibliografía, el proceso de innovación y cambio en la empresa familiar, que se construye sobre la base de los valores y el conocimiento que se transmite de generación en generación, puede verse dificultado en ocasiones por una serie de aspectos, que limitan o incluso impiden la implantación del cambio en la organización (Rumelt, 1995; Klein y Sorra, 1996). Rumelt (1995) desarrolla un modelo de cinco fuerzas explicando estas cuestiones que pueden obstaculizar la implantación de un proceso de cambio en la organización.

La interacción entre empresa y familia puede suponer un obstáculo para la capacidad de renovación y emprendimiento en la empresa familiar (Webb, Ketchen e Ireland, 2010). Esto puede deberse a, por una parte, cierta homogeneidad de pensamiento, la falta de debate y de aportación de ideas diferentes en el seno de la familia puede dificultar la aparición de nuevas propuestas. Por otra parte, un sentido profundo y arraigado de identidad familiar puede ser una fuente de inercias, si limita la renovación y la amplitud de perspectivas que permitan la adaptación de su actividad empresarial, acorde con las nuevas exigencias de la competencia.

El desarrollo e implantación de los procesos $\mathrm{y}$ actividades de innovación implica una serie de cambios en el seno de la organización, por tanto no es un proceso sencillo y plantea problemas específicos que conviene considerar para asegurar, en la medida de lo posible, que la organización alcance las ventajas derivadas de la adopción de la novedad a introducir. La decisión de adoptar una innovación no garantiza por sí misma una correcta implantación mediante su conversión en una rutina más de la organización (Lorenzo, 2003), existiendo una serie de obstáculos a la innovación que pueden retrasar, limitar e incluso impedir la implantación de los cambios necesarios en la organización.

En el apartado siguiente, se hará una breve exposición del modelo de las cinco fuerzas de 
inercia de Rumelt (1995), que ofrece una explicación de los obstáculos que se puede encontrar una organización para iniciar e implantar el cambio. Este modelo se aplica a las características específicas de las empresas de propiedad familiar como una aproximación al estudio de las inercias que obstaculizan la innovación en la empresa familiar.

\section{Aplicación del modelo de inercias de Rumelt a la empresa familiar}

\subsection{El modelo de Rumelt}

A pesar de las premisas de la teoría económica, que suponen la flexibilidad de las empresas, la racionalidad de la acción colectiva y la homogeneidad de las creencias entre las empresas de un mismo sector, las empresas no siempre reaccionan con prontitud a los cambios en el entorno (Lorenzo, 2003). Cambiar la estrategia, la estructura y los procedimientos organizativos es difícil, costoso, arriesgado y requiere el necesario tiempo para su implantación (Rumelt, 1995). Así, Klein y Sorra (1996) indican que la implantación de los cambios consiste, en última instancia, en cambiar la conducta de las personas, y que esto depende del ajuste con los valores de las personas y del clima de la implantación.

La falta de flexibilidad y de disposición al cambio se denomina inercia y se define como la persistencia firme de las formas y funciones actuales (Rumelt, 1995), que si son eficientes, dan lugar a inercia beneficiosa $y$ en caso contrario, suponen un problema a resolver. Una evidencia clara de los efectos de la inercia en las organizaciones es la persistencia de formas y prácticas ineficaces. Nelson y Winter (1982) consideran que las habilidades y capacidades de las organizaciones se basan en sus rutinas para realizar tareas, por lo que la inercia sería el estado natural de la organización, puesto que las empresas sólo pueden hacer aquello para lo que han desarrollado rutinas, así pues, si no hay una rutina para realizar una tarea nueva, ésta no se puede ejecutar (Lorenzo, 2003).
El modelo propuesto por Rumelt (1995) considera cinco fuentes básicas de inercia: percepción distorsionada, falta de motivación, falta de respuesta creativa, barreras políticas y acciones desconexas. Estas cinco fuentes básicas o fricciones actúan de forma escalonada, de modo que cada una de ellas representa un nivel de inercia, cuya superación lleva al escalón siguiente.

La primera fuente de inercia se encuentra en la percepción. $\mathrm{Si}$ no se interpretan correctamente las señales emitidas dentro y fuera de la organización que indican la necesidad o la oportunidad de innovar, es evidente que no se pondrá en marcha tal innovación. Si se supera esta primera barrera de la percepción, puede ser que no se produzca el cambio por falta de motivación para el mismo. Es decir, que no se encuentren alicientes suficientes para la innovación (Lorenzo, 2003), que no se vean las ventajas que conllevaría el proceso de cambio.

Si existen razones suficientes para crear y desarrollar la innovación, la siguiente fuente de inercia puede encontrarse en su falta de respuesta creativa ocasionando que la dirección de la empresa no encuentre el camino adecuado a seguir. Esto es, se asume la necesidad de innovación y se considera que esta innovación tendrá más ventajas que inconvenientes, pero no se sabe cuál es la dirección adecuada a seguir para desarrollar el proceso. En el caso de superar los obstáculos anteriores, pudiera ser que no se emprenda un proceso de cambio debido a la existencia de barreras organizativas internas que lo condicionen, como pueden ser las diferencias y rivalidades existentes entre los distintos departamentos y unidades organizativas de la empresa. Por último, si se vence esta resistencia interna, la falta de coherencia en las acciones podría también poner en peligro el éxito del proyecto de innovación.

\subsection{Aplicación a la empresa familiar y proposiciones de investigación}

La empresa familiar presenta unas características únicas por la influencia de la familia en la empresa y la interacción entre los

Lorenzo Gómez, J.D. y Núñez-Cacho Utrilla, P. (2012). Inercia e innovación en la empresa familiar: una primera aproximación. Revista de Empresa Familiar, 2(2), 23-40. 
familiares a nivel individual y el negocio, lo que generará sus propios obstáculos o inercias a la innovación. $\mathrm{Su}$ identificación facilitará la mejora de la actitud innovadora de la empresa, por ello, a continuación se analizarán con mayor profundidad las cinco fricciones del modelo de Rumelt (1995) aplicadas al caso de la empresa familiar, para posteriormente formular una serie de proposiciones que servirán como base de un análisis más preciso de la influencia que tienen las fuentes de inercia en los procesos de innovación en la empresa familiar.

\subsubsection{Percepción distorsionada}

Un proceso de cambio se inicia a partir de la percepción de la necesidad del mismo. La percepción incorrecta de las señales que anticipan el cambio puede impedirlo, y esto puede suceder por tres razones: la miopía, la autosuficiencia y el pensamiento cuadriculado (Rumelt, 1995).

La miopía se refiere a la incapacidad de la empresa para ver el futuro con claridad. Una fuente de miopía es la rotación frecuente de directivos, que impide que se planteen objetivos a largo plazo por las escasas expectativas de ver sus frutos. También puede tener su origen en un énfasis excesivo en los objetivos a corto plazo que sirven como base para la evaluación de las personas. En la empresa familiar, la miopía puede tener su origen en la dualidad de roles familiares $\mathrm{y}$ directivos que desempeñan las personas, que puede afectar negativamente al reconocimiento y la percepción de oportunidades, generando ansiedad y confusión normativa que lleva a mezclar cuestiones familiares y empresariales (Tagiuri y Davis, 1996).

Los problemas en la percepción correcta también se pueden derivar del rechazo a toda información que sea contraria a lo que se espera o se desea, bien sea por autosuficiencia o por miedo. La autocomplacencia en los éxitos pasados puede impedir una correcta apreciación de las nuevas amenazas y oportunidades del entorno. Puede ocurrir que la empresa familiar no perciba las señales para el cambio porque existan problemas de comunicación u obstáculos al intercambio de conocimiento dentro de la familia (Zahra, Neubaum y Larrañeta, 2006), de manera que al no disponer de la información completa no se aprecien esos mensajes de cambio. La visión de la empresa basada en el conocimiento (Kogut y Zander, 1992; Grant, 1996) ha centrado su atención sobre la importancia de las fuentes de conocimiento en el éxito de la innovación (Bigliardi y Dormio, 2009).

Por otro lado, la empresa familiar presenta un complejo equilibrio entre empleados familiares y no familiares. En este contexto, aparece el nepotismo cuando el baremo o los criterios de cualquier índole, utilizados para valorar, promocionar y retribuir a los miembros de la familia propietaria, son diferentes a los aplicados a los empleados no familiares. El nepotismo se vincula con la percepción de justicia por parte de los empleados no familiares, de manera que cuando éstos están vinculados a actividades de exploración previa a la implantación de innovaciones y están preocupados por la justicia procedimental y distributiva, es decir, por el modo en que se recompensarán los resultados de sus trabajos, la transmisión del conocimiento generado puede verse afectada (Webb et al., 2010). Además, aquellas organizaciones caracterizadas por el nepotismo se convierten en poco atractivas para nuevos profesionales externos, perdiéndose la posibilidad de aportar nuevas ideas y puntos de vista. Por otro lado, se complica la retención del talento existente ya que, al no percibir justicia en las decisiones, los trabajadores no familiares buscarán otras oportunidades fuera de la empresa. Finalmente, al elegir a familiares para cubrir vacantes de la organización se corre el riesgo de promocionar a puestos de responsabilidad a personas poco cualificadas, por el mero hecho de ser familiares (Kets de Vries, 2003).

Una tercera forma de percepción incorrecta o distorsionada se puede denominar pensamiento cuadriculado y se manifiesta de tres formas distintas: por la uniformidad de pensamiento impuesta por los grupos dentro de la organización; por los actos reflejos mentales provocados por los modelos de pensamiento 
imperantes; y por la tendencia a la equiparación de una situación actual a una anterior, que lleva a repetir las actuaciones anteriores, aunque se trate de problemas distintos en contextos diferentes, que no admiten una solución única (Rumelt, 1995; Lorenzo, 2003).

En el caso de las empresas familiares, un aspecto que puede contribuir a la percepción distorsionada es el paternalismo, entendido como es la protección excesiva hacia las personas hasta el punto de interferir en sus decisiones y autonomía (Chirico y Nordqvist, 2010). Los propietarios tienden a proteger a los empleados familiares, mientras que les deniegan responsabilidades y la libertad para expresar sus ideas y tomar decisiones de manera autónoma promoviendo esta inercia familiar (Dyer, 1986). Generalmente, el paternalismo es más común en la primera generación (Schein, 1983) denominando Davis y Harveston (1988) "sombra generacional" a la persistencia de modelos de negocio previos a lo largo de la evolución de la empresa, causada por una influencia excesiva del fundador. El paternalismo se puede relacionar también con la homogeneidad de pensamiento (Webb et al., 2010) que ocurre cuando la influencia del fundador sobre el resto de los miembros de la familia propicia que todos tengan una disposición semejante ante los cambios del entorno y que perciban las mismas señales (Miller y Le Breton-Miller, 2006). Todo esto se vincula con una "endogamia" que puede estar afectada por la escasa formación y experiencia fuera del ámbito del negocio familiar del trabajador miembro de la familia, pues como señala Koellinger (2008) el bajo nivel formativo actuará como freno a la innovación.

Como se ha puesto de manifiesto, cabe esperar que una percepción distorsionada de las señales del cambio afecte negativamente a la capacidad innovadora de la empresa familiar, lo que podría ser objeto de análisis de forma más concreta en las siguientes proposiciones:

P.1.1. La dualidad de roles -familiares y empresariales- de los empleados familiares potencia la percepción distorsionada $\mathrm{y}$, en consecuencia, restringe la capacidad de innovación de la empresa.
P.1.2. El nepotismo en la gestión de las empresas familiares afecta negativamente a su capacidad de innovación.

P.1.3. El paternalismo en las empresas familiares afecta negativamente a su capacidad de innovación.

\subsubsection{Falta de motivación}

Si la percepción de la necesidad o de la oportunidad del cambio es correcta, puede ser que no se emprenda el proceso por falta de motivación suficiente. Esto significa que no se aprecien claramente los beneficios concretos de poner en marcha el proceso. Las razones que explican esta fuente de inercia se refieren a los costes directos que conlleva el cambio (inversiones, cambio de proveedores, etc.), o a los costes de canibalización, que se refieren a que el éxito de un producto nuevo cause el declive o la desaparición del producto anterior.

Las empresas familiares se encuentran con los problemas de financiación derivados de su condición familiar, de su limitado acceso a los mercados de capitales por su deseo de continuar manteniendo el control de la empresa. Además puede otorgar prioridad financiera a la familia frente al negocio, lo que llevaría a rechazar inversiones relacionadas con la innovación. Por el contrario, la apertura del capital a fuentes de financiación ajena propicia estrategias más orientadas a la innovación, relacionadas con la capacidad de explorar y adquirir nuevos conocimientos y tecnologías. Cuando entran socios externos en el capital de la empresa familiar para proporcionar una estabilidad financiera a la empresa, ésta se orienta en mayor medida a estrategias de innovación pudiendo destinar recursos a este tipo de proyectos (Tylecote y Visintin, 2007; Pittino y Visintin, 2009).

Por otro lado, cuando la empresa se encuentra inmersa en procesos sucesorios la complejidad e incertidumbre le puede llevar a una actitud más reticente, disminuyendo la motivación de la dirección para llevar a cabo procesos de innovación. Con frecuencia, la falta 
de un sucesor definido y las dudas sobre la continuidad de la empresa familiar llevan a adoptar actitudes conservadoras con respecto a la innovación. También cuando confluyen en puestos directivos simultáneamente miembros de dos generaciones diferentes, la influencia de la generación más veterana puede llevar a estrategias menos innovadoras (Ensley y Pearson, 2005), disminuyendo la motivación que puede aportar la generación más joven.

Distintos factores pueden ocasionar la falta de motivación para la innovación del liderazgo y se relacionan con el deseo de mantener la armonía dentro de la familia. Por un lado, emprender nuevas acciones puede que no compense la pérdida de tiempo destinado a la familia, la unidad y la calidad de las relaciones entre los familiares. De este modo la empresa familiar tiene una menor expectativa de beneficio financiero, prestándole mayor atención a variables socioemocionales (GómezMejía et al., 2007), y encontrándose menos incentivada para llevar a cabo actividades de innovación o tomar decisiones emprendedoras (Meek, Pacheco y York, 2010).

Otra fuente de inercia relativa a la falta de motivación puede ser la dispersión de la propiedad en la empresa familiar. Cuando el capital se encuentra repartido entre numerosos familiares pueden aparecer los problemas clásicos de agencia (valores, objetivos y visión), dando origen a conflictos y perdiéndose la alineación de los intereses de directivos, familiares y accionistas. De este modo, la organización entra en un punto muerto estratégico mayor que en otras empresas que están más concentradas en lo que a propiedad se refiere (Schulze et al., 2001). Finalmente, el miedo a que los cambios afecten a otros familiares o a la reputación de la empresa puede servir como freno a las iniciativas innovadoras, propiciadas por la falta de motivación (Craig y Dibrell, 2006).

Por lo tanto, la falta de motivación para la innovación en la empresa familiar se podría contrastar a partir de las siguientes proposiciones:
P.2.1. La tendencia a la autofinanciación de la empresa familiar reduce su capacidad de innovación.

P.2.2. Las empresas inmersas en procesos sucesorios no emprenden proyectos de innovación.

P.2.3. El temor a que los cambios afecten al status de los miembros de la familia disminuye la motivación para llevar a cabo actividades de innovación.

P.2.4. El temor a que los cambios afecten a la reputación de la empresa familiar disminuirá la motivación para llevar a cabo actividades de innovación.

P.2.5. La dispersión de la propiedad afectará negativamente a la motivación para llevar a cabo actividades innovadoras.

\subsubsection{Falta de respuesta creativa}

Aunque la percepción sea precisa y exista motivación para la innovación, la implantación de ésta podría bloquearse por la falta de una orientación concreta. Es decir, se asume la necesidad de un cambio de rumbo en la organización, pero que no se sabe cuál es el camino adecuado a seguir.

Esta fuente de inercia se puede deber a tres factores principales: la velocidad y complejidad de los cambios en el entorno, que impiden un análisis sosegado de la realidad; la resignación, entendida como la tendencia a considerar que los problemas son naturales e inevitables, y no tienen solución; y una visión estratégica inadecuada, en referencia a la falta de compromiso claro de la dirección con la visión para poder ejercer un liderazgo efectivo (Rumelt, 1995; Lorenzo, 2003).

Una endogamia excesiva, junto con las carencias de formación y experiencia fuera del negocio familiar puede afectar negativamente la orientación emprendedora en la empresa familiar (Koellinger 2008; Chirico y Norqvist, 2010), encontrándose la empresa sin capacidad creativa para responder a las demandas del entorno (Miller y Le Breton-Miller, 2006).

Por otro lado, los conflictos, celos y rivalidades familiares pueden propiciar que los

Lorenzo Gómez, J.D. y Núñez-Cacho Utrilla, P. (2012). Inercia e innovación en la empresa familiar: una primera aproximación. Revista de Empresa Familiar, 2(2), 23-40. 
miembros más veteranos de la organización disminuyan su transmisión de información a la siguiente generación (Lansberg, 1999; Zahra et al., 2009) y puede ocurrir también que algunos miembros de la familia no tengan interés en el negocio $o$ no deseen adquirir nuevos conocimientos (Grote, 2003; Le Breton-Miller et al., 2004). Todo ello llevará a la ausencia de capacidad para emitir una respuesta creativa a los requerimientos de cambio recibidos del entorno.

Finalmente, esta falta de respuesta puede estar propiciada por una visión estratégica inadecuada, causada por obstáculos organizacionales, por la inexperiencia de la generación en el poder o por su escasa cualificación y conocimiento (Bigliardi y Dormio, 2009), lo que ocasiona un liderazgo inadecuado en la organización y puede ser una consecuencia de errores cometidos en la sucesión, bien por la elección equivocada del sucesor inadecuado o bien por cerrar en falso el proceso. Por último, podría aparecer nuevamente el exceso de personalismo, implantándose en la organización la idea de que si el fundador no encuentra el camino, no hay camino (Davis y Harveston, 1999; Webb et al., 2010).

A partir de lo anterior, la falta de respuesta creativa en la empresa familiar se podría contrastar a partir de las siguientes proposiciones:

P.3.1. La escasez de formación y experiencia fuera del negocio familiar influye negativamente en la capacidad innovadora de la empresa familiar.

P.3.2. La falta de comunicación en el entorno de la familia influye sobre la capacidad emprendedora.

P.3.3. La sombra generacional afecta negativamente a la capacidad de innovación en la empresa familiar.

\subsubsection{Barreras políticas y organizativas}

Las principales fuentes de desacuerdo entre las personas son las diferencias en intereses personales, en creencias $y$ en valores fundamentales, que están en la base de los tres tipos de barreras políticas: las políticas departamentales, en referencia a la oposición de los directivos al cambio porque implica lucha de poder, ganadores y perdedores; las creencias irreconciliables entre grupos, que pueden llevar a adoptar una solución de compromiso basada en un consenso artificial, que desvirtúa la innovación propuesta y los valores arraigados, a los que Rumelt (1995) describe como un equivalente del patriotismo a escala de la organización (Lorenzo, 2003).

De acuerdo con el modelo de los tres círculos de Tagiuri y Davis (1996), en la empresa familiar participan tres grupos de interesados, cada uno de ellos con su visión particular. Estos grupos están representados por la propiedad, la familia y la empresa. Las visiones de cada uno de estos grupos, que en principio son diferentes, pueden suponer un obstáculo para la innovación. Una primera consecuencia de estas diferencias en intereses entre los distintos grupos es que pueden actuar como barrera política a la hora de tomar decisiones. Además, el compromiso emocional de los familiares con la empresa puede propiciar la ausencia de objetividad en la comunicación entre los grupos, el resentimiento y hostilidad en las relaciones. De este modo, se pueden generar creencias irreconciliables entre los grupos que actuarían como freno de las iniciativas innovadoras.

Las barreras pueden aparecer también a partir de los conflictos entre trabajadores familiares y no familiares, derivados del nepotismo en la toma de decisiones (Webb et al., 2010) lo cual dificulta la implantación de los procesos de innovación en la empresa familiar y lleva a alcanzar soluciones de compromiso que desvirtúan la esencia del cambio que se quiere implantar.

Otro ejemplo de barreras políticas y organizativas en la empresa familiar son las derivadas de la indefinición estructural y el solapamiento de papeles familiares $y$ empresariales, que son propias de las primeras etapas de la empresa familiar (Gersick, Davies, Hampton y Lansberg, 1997). Asimismo, no es infrecuente que persistan en la empresa familiar ideas que estuvieron vigentes en su momento, 
aunque hayan quedado obsoletas o no se puedan aplicar en la actualidad, y que sin embargo se mantienen como valores arraigados. En ocasiones, los principales defensores de estos valores arraigados no son los familiares que trabajan en la empresa, sino empleados no familiares con una larga trayectoria en la empresa familiar, que mantienen una forma equivocada de fidelidad al fundador o a las generaciones anteriores, lo que supone una fuente de inercia. Este caso suele aparecer cuando una nueva generación toma el relevo al frente de la empresa, manteniendo el equipo que trabajaba bajo la dirección de la generación anterior.

De acuerdo con esto, los obstáculos derivados de las barreras políticas y organizativas en la empresa familiar se podrían sintetizar en las proposiciones siguientes:

P.4.1. Las interacciones entre los distintos grupos de interés de la empresa familiar afectan a su capacidad de innovación.

P.4.2. Los conflictos emocionales afectan negativamente a la capacidad de innovación de la empresa familiar.

P.4.3. La presencia de nepotismo aumenta las barreras políticas como obstáculo en los procesos de innovación.

\subsubsection{Acciones desconexas}

Una vez superadas todas las inercias descritas anteriormente, es posible que aún no se pueda poner en marcha el proceso de cambio. Los motivos principales que pueden bloquear el cambio son: la inacción del liderazgo, debido al temor de los directivos encargados de implantar el cambio de alterar el status quo existente dentro de la organización; las rutinas establecidas, la necesidad de abandonar prácticas establecidas para hacer cosas nuevas y desconocidas, que aporta una gran cantidad de inercia; los problemas de acción colectiva, esto es, la dificultad de movilizar en una dirección homogénea a toda una organización; y la carencia de las capacidades necesarias para afrontar el cambio con garantías de éxito (Rumelt, 1995; Lorenzo, 2003).
En la empresa familiar, el nepotismo puede estar presente en los procesos de contratación y promoción (Padgett y Morris, 2005). Con frecuencia, los nuevos proyectos se ralentizan e incluso se detienen para no alterar el status en la organización de miembros familiares que pueden tener la aspiración de promocionar en un nuevo proyecto, apartando del mismo a empleados no familiares con mayor capacitación, con el consiguiente perjuicio para la percepción de justicia por parte de estos últimos (Barnett y Kellermanns, 2006).

Otro aspecto que puede repercutir en la inacción del liderazgo es la generación en que se encuentre la empresa. Pittino y Visintin (2009) señalan que los fundadores tienen una mayor orientación a la innovación, adoptan una estrategia más prospectiva y analítica que las empresas en segunda generación y siguientes. Los fundadores tienen poder formal e informal para destinar los recursos a explorar ideas innovadoras (Zahra, 2005), mientras que las segundas generaciones y siguientes adoptan una estrategia menos emprendedora, pues tienen más interés en preservar la empresa y en maximizar sus beneficios que en las actividades innovadoras (Ellington et al., 1996; Eddleston, 2008), además de no detentar el poder en la forma absoluta del fundador. También, cuando la empresa se encuentra inmersa en procesos sucesorios, la complejidad e incertidumbre de esta etapa puede derivar en una actitud más conservadora, como consecuencia de la presencia en los puestos directivos de miembros de dos generaciones distintas. La influencia de la generación más veterana puede orientar hacia estrategias más conservadoras (Ensley y Pearson, 2005), por lo que la inacción del nuevo líder aún en fase de consolidación puede estar motivada por un proceso de sucesión inadecuado, en el que se haya cometido errores o que se haya cerrado en falso, propiciando un liderazgo sin las capacidades necesarias para implantar los procesos de innovación de manera adecuada.

En consecuencia, se podría analizar la incidencia de los problemas de acciones desconexas mediante las proposiciones siguientes: 
P.5.1. Primar los intereses familiares por encima de los intereses empresariales afecta negativamente a la capacidad de innovación de la empresa familiar.

P.5.2. Las empresas familiares en segunda o posterior generación presentan mayores inercias derivadas de las acciones inconexas que las empresas de primera generación.

P.5.3. Los procesos de sucesión potencian la aparición de inercias por acciones desconexas.

\section{Discusión y conclusiones}

La innovación en la empresa familiar es un aspecto escasamente estudiado, sobre el que quedan por responder numerosas preguntas. Entre los varios tópicos que aparecen al tratar sobre la empresa familiar, se le suele atribuir un carácter más conservador en comparación con la empresa no familiar, pero no se han aportado argumentos solventes que ratifiquen $o$ desmientan esta idea. En este sentido, se precisa un mayor esfuerzo por parte de los académicos que trabajan sobre esta materia para comprender mejor los aspectos específicos de la innovación en la empresa familiar. Dentro de esta cuestión genérica, uno de los aspectos menos explorados es el relacionado con los inconvenientes que pueden encontrar las empresas a la hora de afrontar el proceso de innovación.

Con la intención de contribuir a cubrir esta laguna en los estudios sobre empresa familiar, en este artículo, partiendo de los planteamientos de la teoría de recursos y capacidades, el enfoque del conocimiento y las capacidades dinámicas, se ha aplicado el modelo de las cinco fuerzas de Rumelt (1995) analizando la influencia de la familia en las cinco inercias que pueden frenar los procesos de innovación. A la vista del análisis realizado, este familiness negativo puede contribuir a la aparición inercias que dificulten la puesta en marcha de los procesos de innovación, tan necesarios para alcanzar una posición competitiva.

El nepotismo es el factor que está relacionado con mayor número de inercias que afectan a los procesos de innovación en las empresas familiares, ya que dificulta una percepción clara de las señales del entorno; en segundo lugar, crea barreras políticas que dificultan el desarrollo de los procesos de innovación y además, propicia acciones inconexas por parte de los directivos de la empresa.

Craig y Moores (2006) exponen la relación entre generación, ciclo de vida de la empresa e innovación, mientras que Pittino y Visintin (2008) señalan que según la generación que estuviera al frente de la empresa se adopta una estrategia u otra frente a la innovación. En este sentido, el análisis efectuado mediante el modelo de Rumelt muestra que, en función de la generación que esté al frente de la organización, pueden aparecer inercias a partir de acciones inconexas. Estas inercias también se ponen de manifiesto en las empresas familiares que están inmersas en procesos sucesorios pues, en esos momentos, la empresa familiar se puede encontrar desmotivada para acometer nuevos procesos de innovación.

El modelo de Rumelt pone de manifiesto que algunos comportamientos concretos del fundador pueden contribuir a la aparición de estas fuerzas inerciales. Concretamente el paternalismo podría llevar a no percibir las señales de cambio que están en el ambiente; por otra parte, el personalismo del fundador procuraría mantener un único criterio de respuesta al cambio en la empresa, propiciando la falta de respuesta creativa por parte de los demás empleados familiares.

Otro factor a destacar es la dualidad empresa - familia, que propicia la aparición de obstáculos a la innovación ocasionando una percepción distorsionada de las señales de cambio. Esto se pone de manifiesto en la presencia de distintos grupos de interés, conforme al modelo de Tagiuri y Davis (1996), con distintos planteamientos e intenciones, que puede propiciar la aparición de barreras políticas que impidan la puesta en marcha de proyectos innovadores. Por otro lado, esta dualidad familia-empresa puede llevar a la búsqueda de armonía dentro de la familia ocasionando falta de respuesta creativa ante las señales de cambio. Este efecto también lo ocasiona la excesiva endogamia provocada por la escasa experiencia 
fuera de la empresa o por la escasa formación de las empleados familiares.

También se ha de destacar el protagonismo de la transmisión de conocimiento en los procesos de innovación. Con frecuencia, esta transmisión puede verse afectada por celos, rivalidades o falta de entendimiento, de modo que se impida el flujo de conocimiento dentro de la empresa, afectando así a la percepción del entorno y disminuyendo la capacidad de respuesta creativa por parte de los empleados afectados. Estas rivalidades, junto a otros conflictos propios de las empresas familiares, pueden llevar a posiciones irreconciliables que finalmente se podrían traducir en poderosas barreras políticas a la innovación.

Finalmente, se ha de destacar el papel de la financiación y su vínculo con la innovación. En este sentido, los problemas de financiación propios de las empresas familiares, derivados de su deseo de preservar la propiedad en manos de la familia, limitando la entrada de capital externo, podrían derivar en falta de motivación para innovar. Igualmente, cuando la propiedad se encuentre muy dispersa, se podría apreciar esta falta de motivación para la innovación.

Una vez vistos los factores que, aplicando el modelo de Rumelt, generan inercias para la innovación en la empresa familiar, se podrían detectar las principales debilidades de la empresa familiar relacionadas con la innovación y causadas por el familiness negativo. Corrigiendo estos puntos débiles, la empresa familiar se encontrará más capacitada para llevar a cabo procesos de innovación, lo que finalmente redundará en alcanzar una posición competitiva sostenible en el mercado.

\section{Limitaciones y futuras líneas de investigación.}

Como consecuencia de este análisis, se han formulado una serie de proposiciones que podrían servir como punto de partida para un estudio más profundo sobre los obstáculos a la innovación en la empresa familiar. La intención de los autores es analizar estas hipótesis en un trabajo empírico con empresas familiares en Andalucía. Este trabajo empírico podría empezar con un estudio de casos múltiple, con varias empresas familiares de diferentes generaciones y con distinto grado de desarrollo en cuánto a sus órganos de gobierno familiares y empresariales. Este análisis exploratorio permitiría mantener algunas de las proposiciones, al tiempo que posiblemente algunas otras fueran descartadas a partir del estudio de casos. Estas proposiciones serían sometidas a un análisis más amplio mediante técnicas de regresión múltiple en una segunda fase del trabajo empírico, a partir de una muestra de empresas familiares representativa.

La realización de un estudio de estas características permitirá conocer y comprender mejor la naturaleza de las inercias que encuentran las empresas familiares para innovar. La mejor comprensión de los obstáculos que dificultan los procesos de innovación facilitará asimismo la búsqueda de soluciones para evitar, o al menos reducir, el impacto negativo de estas inercias, lo que a su vez redundará en una mejor calidad del desarrollo e implantación de innovaciones en la empresa familiar.

Asimismo, sería interesante realizar un estudio comparativo de los efectos de estas inercias entre empresas familiares $y$ no familiares, lo que ayudaría a concretar las diferencias que existen entre unas y otras y a calibrar los efectos del familiness negativo que se ha venido exponiendo. Quizá sea necesario completar estas líneas de investigación efectuando un estudio longitudinal que permita conocer como varían las inercias a lo largo del tiempo y los efectos de las actuaciones tendentes a reducir los factores de inercia por parte de la empresa familiar.

\section{Referencias}

Aldrich, H.E. y Cliff, J.E. (2003). The pervasive effects of family on entrepreneurship: toward a family embeddedness perspective. Journal of Business Venturing, 18, 573-596.

Anderson, A.R., Jack, S.L. y Dodd, S.D. (2005). The Role of Family Members in Entrepreneurial 
Networks: Beyond the Boundaries of the Family Firm. Family Business Review, 18 (2), 135-154.

Aronoff, C.E. (1998). Megatrends in family business. Family Business Review, 11(3), 181-186.

Autio, E y Mustakallio, M. (2003). Family firm internationalization: A model of family firm generational succession and internationalization strategic postures. Paper presented at the Theories of the Family Enterprise Conference, University of Pennsylvania, Philadelphia.

Barnett, T. y Kellermanns, F. (2006). Are We Family and Are We Treated as Family? Nonfamily Employees' Perceptions of Justice in the Family Firm. Entrepreneurship Theory and Practice, 30(6), 837-854.

Barney, J. (1991). Firm Resources and Sustained Competitive Advantage. Journal of Management, 17 (1), 99-120.

Barney, J. B. y Griffin, R. (1992). The management of organizations: Strategy, structure, behavior. Boston: Houghton Mifflin.

Bermejo, M. (2008). Hacia la empresa familiar líder. Una visión práctica y optimista. Prentice Hall.

Bigliardi, B. y Dormio, A.I. (2009). An empirical investigation of innovation determinants in food machinery enterprises. European Journal of Innovation Management, 12(2), 223-242.

Cabrera-Suárez, M. K., De Saa-Pérez, P. y García, D. (2001). The succession process from a resource and knowledge-based view of the family firm. Family Business Review, 14(1), 37-46.

Casado, F. y Rodríguez, M.A. (2009). La RSE en la gran empresa familiar española. Instituto de la Empresa Familiar.

Chirico, F. y Nordqvist, M. (2010). Dynamic capabilities and trans-generational value creation in family firms: The role of organizational culture,.International Small Business Journal, 28(5), 487-504.

Chrisman, J. J., Chua, J. y Litz, R. (2003). A unified systems perspective of family firm performance: An extension and integration. Journal of Business Venturing, 18(4), 467-472.
Chrisman, J. J., Chua, J. y Steier, L. P. (2003). An introduction to theories of family business. Journal of Business Venturing, 18(4), 441-448.

Chua, J., Chrisman, J. J. y Steier, L. P. (2003). Extending the Theoretical Horizons of Family Business Research. Entrepreneurship Theory and Practice, 27 (4), 331-338.

Craig J. B. y Dibrell, C. (2006). The Natural Environment, Innovation, and Firm Performance: A Comparative Study. Family Business Review, 19(4), 275-288.

Craig, J.B. y Moores, K. (2006). A 10-Year Longitudinal Investigation of Strategy, Systems, and Environment on Innovation in Family Firms. Family Business Review, 19 (1), 1-10.

Davis, P. y Harveston, P. (1988). The influence of family on the family business sucession process:a multigenerational perspective. Entrepreneurship Theory and Practice, 22, 31-53.

Debicki, B.J., Matherne, C.F. III, Kellermanns, F.W. y Chrisman, J.J. (2009). Family Business Research in the New Millennium: An Overview of the Who, the Where, the What, and the Why. Family Business Review, 22 (2), 151-166

Drucker, P.F. (1985). The discipline of innovation. Harvard Business Review, 63 (3), 67-72.

Dyer, W. G. (2006). Examining the "Family Effect" on Firm Performance. Family Business Review, 19 (4), 253-273.

Dyer, W.G. (1986). Cultural changes in family business. John Bassey: San Francisco.

Eddleston, K. A. (2008). Commentary: The Prequel to Family Firm Culture and Stewardship: The Leadership Perspective of the Founder. Entrepreneurship Theory and Practice, 32(6). 10551061.

Ellington, E.P., Jones, R.T. y Deane, R. (1996). TQM adoption practices in family owned business. Family Business Review, 9. 5-14.

Ensley, M. D. y Pearson, A. (2005). An Exploratory Comparison of the Behavioral Dynamics of Top Management Teams in Family and Nonfamily New Ventures: Cohesion, Conflict, 
Potency, and Consensus. Entrepreneurship Theory and Practice, 29(3), 267-284.

Erickson, T., Sorheim, R. y Reitam, B. (2003). Family Angels vs. Other Informal Investors. Family Business Review, 3 (3), 163-171.

Gallo, M.A. (1995). Empresa familiar. Texto y casos. Editorial Praxis.

Gersick, K., Davis, J., Hampton, M. y Lansberg S., I. (1997). Life cycles of the family business. Boston: Harvard University Press (Ed.).

Gómez-Mejía, L., Takacs, K., Núñez, M., Jacobson, K. y Moyano, J. (2007). Socioemotional Wealth and Business Risks in Family-controlled Firms: Evidence from Spanish Olive Oil Mills. Administrative Science Quarterly, 52(1), 106-137.

Grant, R.M. (1996). Toward a Knowledge based theory of the firm. Strategic Management Journal, 17, 109-122.

Grant, R.M. (1991). The Resource-Based Theory of Competitive Advantage: Implications for Strategy Formulation. California Management Review, 33 (3), 114-135.

Habbershon, T.G. y Williams, M.L. (1999). A resource-based framework for assessing the strategic advantages of family firms. Family Business Review, $12,1-15$.

Habbershon, T.G., Williams, M.L. y McMillan, I.C. (2003). A unified systems perspective of family firm performance. Journal of Business Venturing, $18,451-465$.

Haynes, J., Walker, R., Rowe, B. y Hong, G. (1999). The intermingling of business and family finances in family owned businesses. Family Business Review, 12, 225-240.

Hoffman, J., Hoelscher, M. y Ritch, S. (2006). Achieving Sustained Competitive Advantage: A Family Capital Theory. Family Business Review, 19(2), 135-145.

Hoy, F. y Verser, T.G. (1994). Emerging Business, Emerging Field: Entrepreneurship and the Family Firm. Entrepreneurship Theory and Practice, 19(1), 9-23.
Kellermanns, F. (2005). Family Firm Resource Management: Commentary and Extensions. Entrepreneurship Theory and Practice, 29(3), 313319.

Kets de Vries, M. (2003). Doing an Alexander: Lessons on Leadership by a Master Conqueror. European Management Journal, 2003, 21(3), 370375.

Klein, K.J. y Sorra, J.S. (1996). The challenge of innovation implementation. Academy of Management Review, 21 (4), 1055-1080.

Koellinger, P. (2008). Why are some entrepreneurs more innovative than others?. Small Business Economics, 31 (1), 21-37.

Kogut, B. y Zander, U. (1992). Knowledge of the firm, combinative capabilities and the replication of technology. Organization Science, 3(3), 383-397.

Lansberg, I. (1999). Succeeding Generations: Realizing the Dream of Families in Business. Boston, MA: Harvard Business School Press.

Larraza, M., Wiseman, R. M., Gómez-Mejía, L. R. y Welbourne, T. M. (2007). Disentangling compensation and employment risks using the behavioral agency model. Strategic Management Journal, 28(10), 1001-1019.

Le Breton-Miller, I., Miller, D. y Lloyd, S. (2004). Toward an Integrative Model of Effective FOB Succession. Entrepreneurship Theory and Practice, 28, 305-328.

Levering, R. y Moskowitz, M. (1993). The 100 Best Companies to Work For in America. (V ed.). New York: V Plume

Lorenzo Gómez, J.D. (2003). El cambio estratégico en las organizaciones. Servicio de Publicaciones de la Universidad de Cádiz.

Meek, W.R., Pacheco, D.F. y York, J.G. (2010). The impact of social norms on entrepreneurial action. Journal of Business Venturing, 25 (5), 496509 .

Miles, R.E. y Snow, C.C. (1978). Organizational Strategy, Structure and Processes. McGraw-Hill, Nueva York. 
Miller, D. L y e Breton-Miller, I. (2006). Family Governance and Firm Performance: Agency, Stewardship, and Capabilities. Family Business Review, 19(1), 73-87.

Moores, K. (2009). Paradigms and Theory Building in the Domain of Business Families. Family Business Review, 22, 167-180.

Nelson, R.R. y Winter, S.G. (1982). An Evolutionary Theory of Economic Change, The Belknap Press of Harvard University Press, Cambridge, Massachusetts.

Nuñez-Cacho, P. y Grande. F. A. (2012). Family businesses: How to measure their performance. African Journal of Business Management, 6(12), 4612-4621.

Padgett, M. Y. y Morris, K. A. (2005). Keeping it 'all in the family': Does nepotism in the hiring process really benefit the beneficiary? Journal of Leadership and Organizational Studies, 11(2), 3445.

Pittino, D. y Visintin, F. (2009). Innovation and strategic types of family SMEs. A test and extension of Miles and Snow's configurational model, Journal of Enterprising Culture, 17, 257-295.

Rogoff, E.G., Kay, R. y Heck, Z. (2003) Editorial: Evolving research in entrepreneurship and family business: recognizing family as the oxygen that feeds the fire of entrepreneurship. Journal of Business Venturing, 18 (5), 559-566.

Rumelt, R.P. (1995). Inertia and transformation. En MONTGOMERY, C.A. (1995). Resource-Based and Evolutionary Theories of the Firm: Towards a Synthesis (pp 101-132). . Kluwer Academic Publishers

Schumpeter, J.A. (1934). The Theory of Economic Development. Cambridge, Massachusetts: Harvard University Press,.

Sciascia, S., Chirico, F. y Mazzola, P. (2010). Entrepreneurial Orientation and Performance in Family-owned Firms: The Role of Family Management. $6^{\text {th }}$ EIASM Workshop on Family Firms Management Research, Barcelona, June 6-8.
Schein, E. (1983). The role of the founder in creating organizational culture. Organizational Dynamics, 12(13): 13-28.

Schulze, W. S., Lubatkin, M. H., Dino, R. N. y Buchholtz, A. (2001). Agency relationships in family firms: Theory and evidence. Organization Science, 12(2), 99-116.

Sharma, P. Chrisman, J.J. y Chua, J.H. (1997). Strategic management of family business: Past research and future challenges. Family Business Review, 10 (1), 1-35.

Sirmon, D. y Hitt, M. A. (2003). Managing resources: Linking unique resources, management, and wealth creation in family firms. Entrepreneurship Theory and Practice, 27(4), 339358.

Steier, L. (2003). Variants of agency contracts in family-financed ventures as a continuum of familial altruistic and market rationalities. Journal of Business Venturing, 18, 597-618.

Steier, L. (2007). New venture creation and organization: A familial sub-narrative. Journal of Business Research, 60, 1099-1107.

Steier, L. (2009). Where Do New Firms Come From? Households, Family Capital, Ethnicity, and the Welfare Mix. Family Business Review, 22 (3), 273-278.

Steier, L. P. (2001). Next-generation entrepreneurs and succesion: an exploratory study of modes and means of managing social capital. Family Business Review, 14(4), 353-367.

Tàpies, J. (2009): Empresa familiar: ni tan pequeña, ni tan joven. Fundación Jesús Serra.

Tagiuri, R. y Davis, J. 1996. Bivalent Attributes of Family Business. Family Business Review, 9(2), 199-208.

Teece, D.J., Pisano, G. y Shuen, A. (1997). Dynamic Capabilities and Strategic Management. Strategic Management Journal, 18 (7), 509-533.

Tokarczyk, J., Hansen, E., Green, M., y Down, J. (2007). A Resource-Based View and Market Orientation Theory Examination of the Role of 
"Familiness" in Family Business Success. Family Business Review, 20(1), 17-31.

Tylecote, A. y Visintin, F. (2007). A New Taxonomy of National Systems of Corporate Governance. En L.Mjøset, T.Clausen (eds.) Capitalisms Compared, Amsterdam: Elsevier JAI.

Van De Ven, A.H., Polley, D.E., Garud, R. y Venkataraman, S. (2001). El viaje de la innovación. El desarrollo de una cultura organizacional para innovar. Oxford University Press México.

Varis, M. y Littunen, H. (2010). Types of innovation, sources of information and performance in entrepreneurial SMEs. European Journal of Innovation Management, 13 (2), 128-154.

Veciana, J.M. (1999). Creación de empresas como programa de investigación científica. Revista Europea de Dirección y Economía de la Empresa, 8 (3), 11-36.

Webb, J., Ketchen, D., y Ireland, R. D. (2010). Strategic entrepreneurship within family-controlled firms: Opportunities and challenges. Journal of Family Business Strategy, 1(2), 67-77.
Wernerfelt, B. (1984). A Resource-based View of the Firm. Strategic Management Journal, 5, 171180.

Zahra, S. A. (2005). Entrepreneurial Risk Taking in Family Firms. Family Business Review, 18 (1), 2340.

Zahra, S. A., Hayton, J. C., y Salvato, C. A. (2004). Entrepreneurship in Family vs. Non-Family Firms: A Resource-Based Analysis of the Effect of Organizational Culture. Entrepreneurship Theory and Practice, 28 (4), 363-381.

Zahra, S., Neubaum, D. y Larrañeta, B. (2007). Knowledge Conversion Capability and the Performance of Corporate and University Spin-offs.. Industrial and Corporate Change. 16, 569-609.

Zahra, S.A., Gedajlovic, E. Neubaum, D. y Schulman, J.M. (2009). A typology of social entrepreneurs: Motives, search processes and ethical challenges. Journal of Business Venturing. 24 (5), 519-532. 